\title{
Médiévales
}

Langues, Textes, Histoire

52 | printemps 2007

Le livre de science, du copiste à l'imprimeur

\section{Charles V, Gervais Chrétien et les manuscrits scientifiques du collège de Maître Gervais}

Jean-Patrice Boudet

\section{(2) OpenEdition}

1 Journals

\section{Édition électronique}

URL : https://journals.openedition.org/medievales/1943

DOI : $10.4000 /$ medievales. 1943

ISSN : $1777-5892$

Éditeur

Presses universitaires de Vincennes

\section{Édition imprimée}

Date de publication : 1 juin 2007

Pagination : 15-38

ISBN : 978-2-84292-202-3

ISSN : 0751-2708

\section{Référence électronique}

Jean-Patrice Boudet, «Charles V, Gervais Chrétien et les manuscrits scientifiques du collège de Maître Gervais ", Médiévales [En ligne], 52 I printemps 2007, mis en ligne le 06 septembre 2009, consulté le 22 avril 2022. URL : http://journals.openedition.org/medievales/1943; DOl : https://doi.org/10.4000/ medievales. 1943 


\section{CHARLES V, GERVAIS CHRÉTIEN \\ ET LES MANUSCRITS SCIENTIFIQUES \\ DU COLLÈGE DE MAÎTRE GERVAIS}

Dans la partie historique de son Elucidaire, composée entre 1494 et 1498 et connue sous le titre de Recueil des plus celebres astrologues, Simon de Phares donne Charles V en modèle à son destinataire, Charles VIII, et il fait grand cas du collège fondé sous le patronage du roi sage par Gervais Chrétien, son premier médecin. Il évoque cette institution et sa bibliothèque à quatre reprises, d'abord dans le prologue :

[...] le tres sage et debonnaire roy Charles le Quint, lequel tant estudia et ayma ceste science de astrologie, en congnoissant et voyant l'utillité d'icelle, fonda et renta a perpetuité ung college et estudians en icelle, en ladicte université de Paris, ainsi qu'il sera veu cy aprés en son ordre, et leur donna belle librarie bien garnie de livres, speres, astrolabes, saphee et autres instrumens servans a ladicte science ${ }^{1}$, qui encores y sont, et mesmement des semblables et pareilz livres que ce detracteur ignorant a voulu calumpnier a tort et sans cause ${ }^{2}$, laquelle fondacion fut confermee par nostre Saint Pere le pappe, lors estant, qui sur ce octroya bulle expresse, par laquelle, entre autres choses, il anathematiza tous ceulx qui presumeroient oster ou diminuer aucuns d'iceulx livres, ce qu'il n'eust esté fait s'ilz eussent esté autres que bons ${ }^{3}$.

1. Sur ces différents instruments, voir E. Poulle, Les Instruments astronomiques du Moyen Âge, Paris, 1983.

2. Le «detracteur ignorant » visé ici par Simon de Phares n'est autre que Guillaume de Rochefort, chancelier de France, qui, en novembre 1490, l'accusa de posséder un « esperit famillier» et fit en sorte qu'un procès lui soit fait comme devin par l'official de Lyon. Sur les circonstances de la composition de l'Elucidaire et la «premiere persecucion » de l'astrologue de Charles VIII, voir Le Recueil des plus celebres astrologues de Simon de Phares, J.-P. BouDET éd., t. II, Présentation et commentaire, Paris, 1999 (Société de l'Histoire de France, 519), p. 85103 .

3. Le Recueil des plus celebres astrologues de Simon de Phares, J.-P. Boudet éd., t. I, Édition critique, Paris, 1997 (Société de l'Histoire de France, 515), p. 22. 
Comme il l'a annoncé dans le prologue, Simon de Phares revient sur ce « colliege de astrologie et medicine » d'une manière plus détaillée dans sa notice sur Charles V:

Il eut en merveilleuse recommandacion les astrologiens et se gouverna par eulx et, par especial, par ung nommé maistre Gervaiz Chrestien, qui fut grant et proffond astrologien et medicin, comme dit est, a la requeste duquel et autres de son sang, aymant ladite science et par grande deliberacion de son grant conseil et de toute l'université de Paris, il voulut construire - et de fait le fist - et ediffier et aprés fonder, ou meilleur lieu de l'université de Paris, ung colliege de astrologie et medicine, ou il mist livres, plusieurs livres singuliers desdites sciences en grant et merveilleux nombre, et telz et semblables livres que la court de Parlement me a renduz et des semblables de ceulx qui sont en differant et que l'on - ses ebethés ignorans - maintient supersticieux contre verité ${ }^{4}$. Y mist aussi plusieurs astralabes, equatoires, speres et autres instrumens, comme saphees, desirees et semblables. Laquelle fondacion il fist confermer par le pappe Urban $\mathrm{V}^{\mathrm{e}}$, ensemble leur donna la disme du village de Caugie qu'il ordonna pour le vivre des deux bourciers qu'il y mist, qui furent appellés escoliers du roy. Fist en oultre anathematizer tous ceulx qui presumeroient oster ou diminuer iceulx livres et instrumens, estans oudit colliege $^{5}$.

Il donne quelques renseignements supplémentaires sur ce qu'il appelle «le colliege du Roy » et sur son fondateur dans la notice qu'il consacre à Maître Gervais :

Maistre Gervais Chrestien, souverain medicin et astrologien, stipendié et moult aprecié du roy Charles le Quint, fut en ce temps en si grande estyme que le roy voulut que sondit colliege eust son propre nom, pour ce qu'il avoist esté l'inventeur et promoteur d'icelle fondacion. Cestui predist la mort du roy Jehan, qui fut le VIII ${ }^{\mathrm{e}}$ jour de avril mil IIICLXIIII, a Londres en Angleterre. Le colliege du Roy est a Paris, entre la rue de la Herpe et Saint Severin, ou milieu de la rue de la Parcheminerie, tirant vers la conservacion, et sont diz les bourciers du coliege de astrologie et medicine, escoliers du roy ${ }^{6}$.

Enfin, une notice sur l'astrologue arabe Albubater donne des détails intéressants sur la bibliothèque de ce collège, que Simon de Phares qualifie de «librairie de astrologie» et qu'il dit avoir fréquentée personnellement:

4. Les «ebethés ignorans » dont il s'agit sont les membres de la faculté de théologie de l'université de Paris qui, par un avis du 19 février 1494, ont condamné comme superstitieux onze volumes ayant appartenu à Simon de Phares, qui lui avaient été confisqués en 1490 par l'official de Lyon. C'est notamment pour récupérer ces volumes que Simon de Phares avait fait appel au parlement de Paris. Ce dernier rejeta finalement l'appel et condamna Simon aux dépens par arrêt du 26 mars 1494, mais l'astrologue avait récupéré entre temps, par arrêt du Parlement du 23 avril 1493, non pas ses fameux onze volumes, mais d'autres livres qui lui avaient été dérobés dans son hôtel lyonnais par un certain Benoît Meslier : voir Le Recueil, op. cit., t. II, p. 104-120.

5. Le Recueil, op. cit., t. I., p. 504-505.

6. Ibid., p. 492-493. 


\begin{abstract}
Albubater, le tres cler philozophe et souverain astrologien fut, selon aucuns, en ce temps. Cestui monstra les subtilles reigles de astrologie que l'on doit investiguer es nativités. Cestui a escript particulierement sur icelles nativités et commence son livre Dixit Albubater et procede par les XII maisons, lequel livre ay veu au colliege du roy Charles le Quint, en la librairie de astrologie a Paris, jouxte le Livre des IX juges, bien correct et approuvé et, par ce, incathené et en usage a qui en veult ${ }^{7}$.
\end{abstract}

Dans un article publié en 1976, Richard Lemay a tiré parti du témoignage de Simon de Phares pour émettre l'hypothèse selon laquelle "l'astronomie, très probablement dans son orientation astrologique, constituait, au XIV siècle, une préoccupation centrale pour les étudiants en arts et en médecine et pour les théologiens du studium de Paris », et que c'était "probablement sous la pression des médecins que l'enseignement de cette discipline reçut le soutien spécial de la Couronne, de l'Université et de la Papauté » ${ }^{8}$. Guy Beaujouan, Danielle Jacquart et moi-même avons eu l'occasion d'émettre quelques réserves à l'égard de cette hypothèse ${ }^{9}$. Mais ma participation à la soutenance de la thèse de Karine Rebmeister-Klein sur les bibliothèques des collèges parisiens à la fin du Moyen Âge, qui comprend une précieuse étude sur la «librairie» du collège de Maître Gervais ${ }^{10}$, m'a incité à consulter l'ensemble des documents disponibles afin d'approfondir la question et de tenter d'y voir plus clair sur les motivations de Gervais Chrétien, sur la place des sciences dans l'institution fondée par lui et sur la diffusion des savoirs scientifiques à Paris à la fin du Moyen Âge.

7. Ibid., p. 291-292. Les deux ouvrages en question sont, d'une part, le De nativitatibus d'Albubater (Abû Bakr al-Hasan ibn al-Khâsib), traduit de l'arabe en latin par Salio de Padoue en 1218 (voir F. J. CARMODY, Arabic Astronomical and Astrological Sciences in Latin Translations. A critical Bibliography, Berkeley-Los Angeles, 1956, p. 136-137, et J.-C. VADET, «Ibn al-Khâsib », Encyclopédie de l'islam, $2^{\mathrm{e}}$ éd., Leyde-Paris, 1975, t. III, p. 859), et, d'autre part, le Liber novem judicum, une compilation sur les interrogations astrologiques attribuée à neuf auteurs différents, traduite de l'arabe en latin au XII ${ }^{\mathrm{e}}$ siècle et du latin en français en 1361, à la demande du dauphin Charles, par Robert Godefroy. L'exemplaire de présentation de ce Livre des neuf anciens juges d'astrologie est le ms. Bruxelles, Bibl. royale 10319, qui a appartenu ensuite aux ducs de Bourgogne, et une autre copie de la fin du XIv ${ }^{\mathrm{e}}$ siècle est le ms. Paris, Bibl. de l'Arsenal 2872, $\mathrm{f}^{\circ} 85 \mathrm{r}^{\circ} \mathrm{a}-309 \mathrm{r}^{\circ} \mathrm{b}$.

8. R. Lemay, «The Teaching of Astronomy in Medieval Universities, principally at Paris in the Fourteenth Century », Manuscripta, n 20 (1976), p. 197-217 (citation p. 204).

9. G. Beaujouan, «Le quadrivium et la Faculté des arts », dans L'Enseignement des disciplines à la Faculté des arts (Paris et Oxford, XIII ${ }^{e}-X V^{e}$ s.), Actes du colloque international, O. Weijers et L. Holtz éd., Turnhout (Studia Artistarum, 4), 1997, p. 185-194 (notamment p. 193-194) ; D. JACQUART, La Médecine médiévale dans le cadre parisien, XIV ${ }^{e}-X V^{e}$ siècle, Paris, 1998, p. 448-465 (en particulier p. 453-457); Le Recueil, op. cit., t. II, p. 248-254.

10. K. RebmeIster-KLein, Les Livres des petits collèges à Paris aux XIV et $X V^{e}$ siècles, thèse de l'université Paris I sous la dir. de Jean-Philippe Genet, 3 vol. dactyl., 2005, dont une version remaniée doit paraitre chez Brill. Sur le collège de Maitre Gervais, voir notamment t. I, p. 153-154, 167, 199 ; t. II, p. 684-701 (éd. des sources), et t. III, p. 1087-1112 (ex-libris, colophons et notices de manuscrits). 


\section{Gervais Chrétien et la fondation du «colliege du Roy »}

Revenons d'abord sur la personnalité du fondateur ${ }^{11}$. Gervais Chrétien, issu d'une famille de petite noblesse normande, semble être né à Vendes (Calvados), dans les années 1320. Boursier du roi en 1345, il étudie les arts et la médecine à Paris, accède à la maîtrise dans cette dernière discipline en 1352 au plus tard, et devient «physicien » de Jean II, puis premier médecin de Charles V. Élu, en 1359, doyen de la faculté de médecine de Paris, il siège ensuite parmi ses maîtres régents, au moins jusqu'en 1379, et collectionne les prébendes canoniales : chanoine du Saint-Sépulcre de Caen, de Notre-Dame de Bayeux, de Saint-Liphard de Meung-sur-Loire (1362), de l'église de SaintQuentin (1364), de Notre-Dame de Rouen (1367), puis de Paris (décembre 1370), il meurt à Bayeux, le 10 mai 1382, où il est enterré dans la cathédrale.

En 1349, maître Robert Clément avait tenté de créer à Paris un nouveau collège de Bayeux, distinct de l'établissement du même nom qui avait été fondé en 1308 par l'évêque Guillaume Bonnet. Situé rue Hautefeuille, au «Pot d'étain », ce collège survivait à peine, alors que le médecin du roi, compatriote normand de Maître Robert, avait acheté, depuis 1360, plusieurs immeubles et rentes immobilières au Quartier Latin ${ }^{12}$. À la requête de la nation normande, le conseil de l'Université décida, le 3 juin 1370, que faute de fonds, les étudiants pris en charge par Robert Clément seraient installés dans un autre collège. Maître Gervais accepta la fusion, le 22 septembre $1370^{13}$, et au total, six maisons ou parties de maisons furent affectées au nouveau collège, placé sous le patronage de Notre-Dame de Bayeux ${ }^{14}$. L'acte officiel de fondation, dressé le 20 février 1371 (n.s.) sous l'autorité du prévôt de Paris, spécifiait que

Maistre Gervaise Crestian, maistre es arts et en medecine en l'université de Paris, premier phisicien de tres excellent et tres crestien prince Charles, par la grace de Dieu roy de France, nostre dit seigneur, afferma et dist en bonne verité que comme ja pieça, il eust propos, devocion et entencion de fonder et creer escoliers a Paris, estudians en la faculté de theologie, du diocese de Bayeux en Normandie [...], des pieça avoit fondé et encores par la

11. Voir S. Le Paulmier, « Notice sur Gervais Chrétien, fondateur du collège de Maître Gervais, et sur Guy Chrétien, trésorier de France », Revue catholique de Normandie, t. 7 (1897), p. 40-60 et 97-129 ; E. WICKERSHEIMER, Dictionnaire biographique des médecins en France au Moyen Âge, Paris, 1936, nouv. éd. sous la dir. de G. Beaujoun, Genève, 1979, t. I, p. 189-190.

12. Voir, entre autres : Paris, Arch. nat., M 163, pièce $n^{\circ} 2$ : vente d'une maison à Paris, rue Saint-Jacques, par Jean Saquary, bourgeois de Paris, à $\mathrm{M}^{\mathrm{e}}$ Gervais Chrétien, maître ès arts et en médecine, 19 mars 1360 (n.s.) ; M 163, pièce $n^{\circ} 6$ : Guillaume Mignon vend à $\mathbf{M}^{\mathrm{e}}$ Gervais Chrétien, « fisicien du roi », 20 sous parisis de rente sur une maison de la rue des Parcheminiers, 18 avril 1366 ; etc. Le détail des immeubles du collège de Maitre Gervais est notamment donné dans l'acte de fondation de février 1371: voir infra, notes 14 et 15 .

13. Paris, Arch. nat., M 167, pièce ${ }^{\circ} 6$.

14. Paris, Arch. nat., $M M 400, \mathrm{f}^{\circ} 15 \mathrm{v}^{\circ}-16$ : inventaire des titres et papiers du collège de Notre-Dame de Bayeux, autrement dit de $\mathrm{M}^{\mathrm{e}}$ Gervais (XVIII ${ }^{\mathrm{e}} \mathrm{s}$.). 
teneur de ces presentes lettres fonde, cree et institue a Paris, en la rue Erembourc de Brie, autrement dite la rue aux Illumineurs, emprés la grant rue Sainct-Jacques, un colleige d'escoliers qui soient du dit diocese, estudians en la science et faculté de theologie, ouquel colleige il aura aucuns escoliers, maistres ou licenciez es ars, continuelment estudians en la dite faculté de theologie, qui prendront pour chascune sepmainne chascun cinq sols parisis pour leurs bourses, pour tant de temps comme il sera dit et ordené par les estatus qui sont ou seront fais sur ce. Et pour ce que la science des ars est proffitable et necessaire a la dite science et faculté de theologie, il y aura oudit colleige aucuns autres escoliers, estudians en la science et faculté des ars, lesquelz prendront chascun trois sols parisis pour bourse, pour chascune sepmainne, par tant de temps comme il sera dit et ordené es dits status. [...] Et pour ce qu'il y met les dis escoliers es ars pour venir et monter a estudier en theologie principalment, et pour aidier a continuer les dis estudians en ladite science et faculté de theologie pour la cause dessusdite, il veut et entent que ce soit tout un colleige de theologie, supposé qu'ils demeurent et habitent en deux maisons prochaines ${ }^{15}$.

Voilà qui est parfaitement clair et qui entre en totale contradiction avec le plaidoyer pro domo de Simon de Phares ! À l'origine, ce «colleige de theologie » comprend en fait dix boursiers : quatre étudiants à la faculté de théologie, originaires du diocèse de Bayeux ou, à défaut, d'un autre diocèse, le plus proche possible, perçoivent une bourse de cinq sous parisis par semaine ; six étudiants ès arts, originaires de Vendes ou de Bayeux, touchent trois sols par semaine. Il n'y a donc, au départ, ni médecins, ni «astrologiens » dans le collège de Gervais Chrétien, dont la structure semble calquée sur le projet de Robert Clément.

Le 13 mars 1371, la toute nouvelle institution bénéficie de la fondation «par feu Maistre Henry Vacaire, jadis physicien de Madame la Royne Jehanne » de deux bourses destinées l'une à un prêtre, étudiant en théologie, l'autre à un artien, ce qui porte à douze le nombre des boursiers ${ }^{16}$. On trouve dans la liste des six « escolliers » mentionnés parmi les membres du collège, à cette date, le nom de Martin Gazel, qui étudia pendant deux ans la théologie avant de profiter lui-même de l'une des deux bourses de médecine établies par Gervais Chrétien en 1377, et qui devint par la suite maître régent de la Faculté et médecin de Charles VI ${ }^{17}$. Les exécuteurs testamentaires d'Henri

15. Paris, Arch. nat., M 163, pièce $\mathrm{n}^{\circ} 8$.

16. Paris, Arch. nat., M 167, pièce $n^{\circ} 9$, et MM 400, $\mathrm{f}^{\mathrm{o}}$ 29. La reine en question est Jeanne de Bourgogne, veuve de Philippe VI. Henri Vacaire, alias Henri Hensey, était lui aussi originaire du diocèse de Bayeux. Maître ès arts et en médecine de l'université de Paris, on le repère comme «physicien» de l'archevêque de Rouen, en 1350-51: voir E. WicKERSHEIMER, Dictionnaire, op. cit., t. I, p. 279.

17. «Honnorable et discrete personne maistre Gervaise Crestien, phisicien du roi nostre dit seigneur», agit en l'occurrence « ou nom et pour mestre Pierre Verel, principal, Jehan Godeffroy, Colin Lasnier, Jehan Mauchaiz, Martin Gazel et Jehan de Vindehagne, tous escolliers...». Sur Martin Gazel, voir E. WiCKERSHEIMER, Dictionnaire, op. cit., t. II, p. 541-542, et Supplément par D. JaCQuART, Genève, 1979, p. 207 ; B. GuenéE, La Folie de Charles VI, Roi Bien-Aimé, Paris, 2004, p. 107, 109, 122, 124, 127-128. 
Vacaire lèguent à cette occasion au collège un certain nombre de biens et d'objets précieux, dont un missel noté à l'usage de Paris et « un livre de philosophie contenant trente-sept livres de philozofie ou environ, pour et au proufit de tous les escolliers dudit hostel ». Un an plus tard, le 6 mai 1372, le roi leur donne les volumes de philosophie et de médecine provenant de la succession de Jean de Pantalye, son chirurgien, prisés à 40 livres parisis ${ }^{18}$.

Charles V multiplie par la suite les gestes de bienveillance en faveur du collège et de son fondateur. Dans le premier inventaire de la «librairie » royale, dressé par Gilles Malet, en 1373, sont mentionnés un exemplaire des «Ethiques glosees, couvert a II hez et II fermoirs »-peut-être la toute récente traduction par Nicole Oresme de l'ouvrage d'Aristote, datée de 1372 -, « donné aus escoliers maistre Gervaise », et un autre volume des «Ethiquez, couvert de cuir noir, a IIII fermoirs », « donné par le roy a maistre Gervese ${ }^{19}$. En 1374, le roi lègue par testament à « l'ostel collegial des escolliers, fondé de nouvel, de maistre Gervais Chrestien », 50 livres de rente pour douze messes solennelles par an qui devront être chantées à sa mémoire. L'année suivante, il donne un reliquaire en vermeil à la chapelle du collège ${ }^{20}$.

Charles V fait également en sorte d'accroître le nombre des boursiers et d'obtenir pour ce faire le soutien à la fois financier et spirituel des autorités religieuses. Le pape Grégoire XI, par bulle du 10 juin 1373, l'évêque de Paris, par lettres du 27 juin 1374, et le roi, par lettres du 10 octobre 1374, confirment la donation des dîmes de Senneville (Seine-Maritime) pour deux nouveaux boursiers en théologie ${ }^{21}$. Le 4 novembre 1373, l'abbé du Mont Saint-Michel associe le collège de Maître Gervais aux prières de son couvent; le 30 août 1377, Grégoire XI (et non pas Urbain V, comme le dit Simon de Phares) confirme la fondation du collège, décision suivie par celle de l'évêque de Paris, Aimery de Maignac, qui, le 15 octobre de la même année, confirme la nomination faite par Gervais de deux boursiers en médecine ${ }^{22}$. Quinze jours plus tard, le 30 octobre 1377, Charles V ordonne

18. Paris, Arch. nat., M 163, pièce $\mathrm{n}^{\circ} 11$, document publié par G. BRUNEL, Images du pouvoir royal. Les chartes décorées des Archives nationales, XIII ${ }^{-}$-XV $V^{e}$ siècle, Paris, 2005, p. 184185. Chirurgien de Philippe VI, Jean II et Charles V, Jean de Pantalye fut chargé de l'embaumement du premier roi Valois en 1350 : voir E. WiCKERSHEIMER, Dictionnaire, op. cit., t. II, p. 459.

19. L. DelisLe, Recherches sur la librairie de Charles V, Paris, 1907, vol. I, p. 81, et vol. II, p. $81, \mathrm{n}^{\text {os }} 479$ et 478 . Le premier cité pourrait à la rigueur être identifiable avec le ms. Paris, Bibl. de l'Arsenal 2668 : voir. K. ReBMEISTER-KLein, Les livres des petits collèges, op. cit., t. III, p. 1088.

20. S. Le Paulmier, « Notice sur Gervais Chrétien», loc. cit., p. 49.

21. Du Boulay, Historia Universitatis Parisiensis, Paris, 1668, réimpr. Francfort-sur-leMain, 1966, t. IV, p. 430-435 ; P. de LoNGuemare, « Le collège de maître Gervais Chrétien à Paris », Bulletin de la Société des antiquaires de Normandie, t. 31 (1916), p. 182-329 (aux p. 203-204).

22. Paris, Arch. nat., M 163, pièces $\mathrm{n}^{\text {os }} 15,19$ et 17. La confirmation de l'évêque de Paris (pièce $\mathrm{n}^{\circ} 17$ ) concerne en effet plus particulièrement la nomination de deux boursiers en médecine, Jean de Troismances, sous-diacre de Bayeux, maître ès arts, bachelier en médecine et curé de la paroisse Saint-Sauveur de Caen, et Martin «Gazelliez» (i.e. Gazel), lui aussi sous-diacre 
d'allouer aux comptes de son trésorier, François Chanteprime, 920 francs d'or, « de laquelle somme nous avons paié certaine rente que nous avons fait acheter pour le vivre de deux estudians fonder ou college de noz escoliers qui demourent en l'ostel de nostre amé et feal phisicien maistre Gervaise Chrestien, lesquels nous avons ordené que ils liront les sciences mathematiques licites et permises a lire en nostre université de Paris ${ }^{23}$.

Cette décision n'est pas isolée : elle va de pair avec la fondation des deux bourses en médecine, qui participe à une tentative de rééquilibrage en faveur d'une discipline pour laquelle seuls trois autres collèges parisiens fournissaient des bourses avant l'avènement de Charles V - ceux de Bayeux (statuts de 1308/15), de Narbonne (1343) et de Justice (1354) -, la fondation de bourses de médecine au collège de Laon, en 1365, par Jean de Coucy, ancien médecin de Jean II, et celle de Jean de Guiscry, autre médecin de Charles V, au collège de Cornouaille, en 1379, s'inscrivant dans la même perspective que celle de Gervais Chrétien ${ }^{24}$. Mais cette décision d'octobre 1377 doit aussi être placée dans le contexte de l'histoire du collège de Maître Gervais à la fin du règne du roi sage pour que l'on puisse en préciser la portée. Les statuts de cette institution sont promulgués en 1378 et dûment approuvés par des lettres de l'évêque de Paris, le 5 mai $1378^{25}$, et par une bulle de Clément VII, le 30 juin $1379^{26}$. En juillet 1378, les exécuteurs testamentaires de Guillaume Baron, chantre de l'église de Bayeux, fondent une bourse supplémentaire pour un étudiant en décret et lèguent au collège une cinquantaine de volumes provenant de sa succession - des livres de droit canon, pour la plupart ${ }^{27}$. En avril 1379, le roi, malade et soucieux de pérenniser la fondation de son premier médecin, accepte solennellement, par lettres patentes, de prendre le titre et l'autorité de fondateur du collège de Notre-Dame de Bayeux, et d'en commettre la direction à son grand aumônier, à qui, après

de Bayeux et maître ès arts. Sur Jean de Troismances, voir E. WiCKERSHEIMER, Dictionnaire, op. cit., t. II, p. 496.

23. L. DesLisle, Mandements et actes divers de Charles V (1364-1380), Paris, 1874, p. $754, \mathrm{n}^{\circ} 1497$.

24. Sur Jean de Coucy $(\dagger 1363)$ et la fondation des bourses de médecine du collège de Laon, voir E. WickersheIMER, Dictionnaire, op. cit., t. I, p. 387-388, Supplément par D. JACQUART, p. 152, et C. FABRIS, Étudier et vivre à Paris au Moyen Âge: le collège de Laon (XIV ${ }^{e}-X V^{e}$ s.), Paris, 2005 (Mémoires et documents de l'École des chartes, 81), p. 78-79 et 322-327. Sur le collège de Cornouaille et la fondation de Jean de Guiscry, voir E. Chatelain, Notice sur un ouvrage de médecine orné de miniatures, copié en 1379, Paris, 1886 ; E. WICKERSHEIMER, Dictionnaire, op. cit., t. I, p. 415-416, et Supplément par D. JACQUART, p. 160.

25. Paris, Arch. nat., M 163, pièce $\mathrm{n}^{\circ} 19$. Ces statuts et la lettre de l'évêque sont édités par P. de Longuemare, «Le collège de maître Gervais Chrétien », loc. cit., p. 263-329. Sans évoquer l'anathème dont parle Simon de Phares, l'évêque promet néanmoins l'excommunication ipso facto à quiconque aliénerait des biens du collège et ferait quelque chose contre ses statuts, quantum ad librarias et librorum conservationem (p. 328).

26. Paris, Arch. nat., M 164, pièces $\mathrm{n}^{\text {os }} 2$, 3 et 4 : vidimus de cette bulle par l'official de Paris et celui de Rouen, 4 et 15 septembre, 20 décembre 1380.

27. Paris, Arch. nat., M 167, pièce $\mathrm{n}^{\circ} 16$. Le catalogue des livres légués par Guillaume Baron est publié par K. ReBMEISTER-KLEIN, Les Livres des petits collèges, op. cit., t. II, p. 686-691. 
la mort du souverain, doivent revenir l'administration et la collation des bourses du collège ${ }^{28}$. De nouveaux statuts, approuvés par l'évêque de Paris en avril 1380, y établissent deux chapelains, et ils sont encore corrigés en janvier 1382: Gervais Chrétien prévoyant que la valeur des dîmes pouvait augmenter, ordonne que le boni annuel serait capitalisé et que lorsqu'il y aurait une somme suffisante, une nouvelle bourse serait créée ${ }^{29}$.

\section{Les motivations de Maître Gervais, celles de Charles V et l'enseignement des «sciences licites»}

De l'ensemble des documents mentionnés plus haut et de quelques autres, il ressort que Simon de Phares n'a pas tort lorsqu'il qualifie l'institution créée par Gervais Chrétien de « colliege du Roy », mais que celui-ci n'a jamais été conçu, dès le règne de Charles $\mathrm{V}$, comme un «colliege de astrologie et medicine ", contrairement à ce qu'il veut nous faire croire.

Le prologue et le premier chapitre des statuts approuvés en mai 1378 donnent les raisons officielles de la création du collège : faire une œuvre pie en contribuant à améliorer l'accès des "pauvres » à l'étude du «trésor des sciences » enseignées à Paris et constituées par les arts libéraux, la théologie et la médecine. Le nombre des boursiers est alors fixé à vingt : douze petits boursiers, étudiants en «arts, logique et philosophie » toucheront désormais 6 sous parisis par semaine pendant les 40 semaines de cours et logeront dans la «maison des artiens»; dix grands boursiers logeront dans la «maison des théologiens » : parmi ces derniers, quatre étudiants en théologie et deux étudiants en médecine toucheront 6 sous parisis par semaine pendant les jours ouvrés et 5 sous hebdomadaires pendant les vacances, alors que deux maîtres ès arts de l'université de Paris, appelés « écoliers du roi » car Charles V

28. Paris, Arch. nat., M 163, pièce $\mathrm{n}^{\circ} 18$ (lettres originales), et JJ 115, fo $166-166 v^{\circ}$ (copie) : «Volo tamen et ordino quod post decessum meum, elemosinarius regis qui pro tempore erit et sui successores, regum elemosinarii, habeant administrationem et collationem bursarum collegii supradicti, prout decet, cum quia idem rex, dominus noster, dictum collegium in redditibus, admortizationibus et libris multipliciter augmentavit, adeo quod sibi debetur principaliter nomen et auctoritas fundatoris et etiam collatoris [...] Dictusque magister Gervasius desideret hoc etiam a nobis instanter et suppliciter fusis precibus, pro gratia et munere speciali postulans obtineri, ut dicti collegii nomen et auctoritatem fundatoris sumere et retinere voluimus. » L'aumônier du roi est alors Pierre de Proverville : voir X. de LA SELLE, Le Service des âmes à la cour. Confesseurs et aumôniers des rois de France du XIII au XV siècle, Paris, 1995 (Mémoires et documents de l'École des chartes, 43), p. 292. Cette décision est à mettre en rapport avec la politique interventionniste de Charles V en la matière : voir N. Gorochov, «Charles V et les collèges parisiens. L'affirmation d'une politique universitaire royale (1364-1380) », dans Paris et ses campagnes sous l'Ancien Régime. Mélanges offerts à Jean Jacquard, Paris, 1994, p. 187-194 ; Th. Kounamé, Le Collège de Dormans-Beauvais à la fin du Moyen Âge. Stratégies politiques et parcours individuels à l'université de Paris (1370-1458), Leyde, 2005 (Education and Society in the Middle Ages and Renaissance, 22), p. 31-38.

29. Paris, Arch. nat., M 164, pièces nos 1,5 et 6 . Voir P. de Longuemare, « Le collège de maître Gervais Chrétien », loc. cit., p. 221. 
a fondé leurs bourses pour qu'ils «lisent les sciences licites », toucheront, outre leur bourse ordinaire de 6 sous par semaine, payée sur toute l'année, le revenu des dîmes du village de Cauchy ${ }^{30}$ - Simon de Phares est bien renseigné sur ce point - jusqu'à la somme de 60 livres tournois. Or, ces bourses, à l'instar de celles des théologiens, sont accordées pour une durée théorique de dix ans, contre six ans seulement aux médecins, ce qui constitue un privilège important ${ }^{31}$.

Ces deux scholares regis sont chargés de dispenser pendant l'ensemble de l'année un enseignement sur «les sciences mathématiques, c'est-à-dire sur les livres d'arts libéraux du quadrivium, jugés licites par les canons sacrés ou en aucune manière réprouvés par l'université de Paris ", l'un à la faculté des arts, les jours prévus pour la lectio, l'autre dans la maison des artiens du collège, les jours de fêtes. Mais ils seront tenus aussi d'assister soit aux cours de médecine de l'Université, soit aux cours de théologie, dans la mesure où leur enseignement spécialisé, considéré comme des lectiones extraordinarie, ne les occupe pas à plein temps ${ }^{32}$. Ces scholares regis sont donc effectivement censés être des spécialistes du quadrivium, mais rien n'indique que leur spécialisation s'appliquait aux autres boursiers, y compris aux étudiants en arts, du moins à titre d'obligation. L'on connait d'ailleurs l'identité de l'un de ces deux «écoliers du roi », en 1379: le rôle des artistes de la nation normande de l'Université indique qu'il s'agissait de Jean Durand, clerc du diocèse de Rouen, étudiant en seconde année en médecine et enseignant en astrologia ex precepto domini regis, qui devint par la suite médecin du duc de Bourgogne Philippe le Hardi ${ }^{33}$.

30. Calvados, cant. d'Isigny.

31. P. de Longuemare, "Le collège de maitre Gervais », loc. cit., p. 272-274. Le nombre total des boursiers y passe à 24 en 1382 (avec sept boursiers théologiens, dont les deux chapelains) et à 26 en 1402, avec la fondation de Guillaume de Lirois, conseiller clerc au Parlement, de deux bourses, l'une de théologien, l'autre d'artien: Arch. nat., M 167, $\mathrm{n}^{\circ} 17$. Mais il est revenu à 24 dans le courant du $\mathrm{Xv}^{\mathrm{e}}$ siècle.

32. Ibid., p. 274-275: «[...] quolibet anno quo continue leget de scientiis supradictis, modo et forma inferius annotatis, et tenebuntur illi duo magistri legere de scientiis mathematicis, videlicet de libros de quadrivio artium liberalium licitos per sacros canones vel per Universitatem Parisiensem nullatenus reprobatos, quorum unus leget in vico Straminum, diebus legibilibus, aliqua hora pro legendo in artium Facultate ordinata, et alter leget de alio libro ejusdem quadrivii in aula artistarum dicti Collegii, diebus festivis et horis in Universitate Parisiensi consuetis [...] Sed, quia non legent ordinarie de dictis scientiis, tenebuntur audire theologiam vel medicinam et quin etiam in domo theologorum et cum theologis et medicis morabuntur et manebunt et ordinabuntur et regentur secundum ordinationes et statute dicte domos... »

33. H. Denifle et E. Chatelain, Chartularium Universitatis Parisiensis, t. III, Paris, 1894, p. 265. Sur ce médecin et astrologue, voir E. WiCKERSHEIMER, Dictionnaire, op. cit., t. I, p. 395, et Supplément par D. JACQUART, p. 154. Je le soupçonne d'être l'auteur des horoscopes de naissance de Marguerite de Flandres, duchesse de Bourgogne, et de son fils Antoine de Bourgogne, né en 1384, conservés sur les gardes du ms. Oxford, Saint John's College 164, f $\mathrm{f}^{\mathrm{o}}$ [IIv ${ }^{\circ}$ ]-[III], ms. qui a appartenu à Charles V et Charles VI et qui comprend notamment L'Espere en françois de Nicole Oresme, le Livre des elections et le traité de l'astrolabe de Pèlerin de Prusse, une traduction française du Liber introductorius d'Alcabitius, ainsi que les horoscopes de Charles V et de ses enfants (voir infra, notes 37 et 39). 
On ignore tout du contenu précis de cet enseignement, mais les leçons de quadrivium des deux scholares regis du collège de Maître Gervais visaient, semble-t-il, à institutionnaliser un apprentissage scientifique semi-privé, dispensé habituellement pendant les jours de fêtes, et l'on peut même se demander, à l'instar de Guy Beaujouan, si ces deux bourses royales n'ont « pas été instituées comme une sorte de contre-feu à l'enseignement semi-clandestin d'une astrologie suspecte ${ }^{34}$. En 1358, en effet, l'Université avait enregistré la requête, formulée par Robert Le Normand, de pouvoir donner des leçons chez lui, à Paris, les jours de fête, post sermonem, sur le Quadripartitum de Ptolémée et le Centiloquium du Pseudo-Ptolémée ${ }^{35}$. L'autorité incontestable de Ptolémée faisait figure ici de paravent et l'on peut supposer que la demande émanant de certains maîtres ès arts devait être forte d'enseigner l'ensemble des doctrines utilisées par les astrologues - les nativités, les révolutions, les élections, les interrogations et les images astrologiques -, et pas seulement les deux premières, qui constituaient les parties de l'astrologie les mieux tolérées par l'Église ${ }^{36}$. Or on remarquera que la fondation des deux bourses de 1377 était assortie d'une réserve qui n'était pas une simple clause de style : les scholares regis «liront les sciences mathematiques licites et permises a lire en nostre université de Paris ». Cette limitation, soigneusement réaffirmée dans les statuts de 1378, paraît exclure la magie, la divination, mais aussi les parties de l'astrologie jugées les plus superstitieuses par les théologiens de l'Université, à savoir les élections et les interrogations, dans lesquelles se délectait justement le dauphin Charles vers $1360^{37}$. L'on peut donc supposer que « les sciences licites » en question se réduisaient aux mathématiques et à l'astronomie proprement dite, voire à l'astrologie des nativités et des révolutions des années, mais ne devaient pas dépasser de beaucoup ces bornes assez étroites. Et l'on aboutit à la conclusion que la fondation de ces deux bourses visait davantage à canaliser les spéculations astrologiques dans une voie autorisée et contrôlée par les théologiens qui dominaient l'université de Paris qu'à une promotion de l'astrologie dans son ensemble. Le fait que Charles $\mathrm{V}$ et Gervais Chrétien aient prévu qu'après la mort du souverain, l'administration et la collation des bourses du collège devaient revenir à l'aumônier du roi va dans le même sens, celui d'un contrôle exercé conjointe-

34. G. Beaujouan, «Le quadrivium et la Faculté des arts », loc. cit., p. 193-194.

35. H. Denifle et E. Chatelain, Auctarium chartularii Universitatis Parisiensis, t. I, Paris, 1894, col. 225. Cette requête est exaucée en même temps que celle d'Albert de Saxe à propos de lectiones sur la Politique d'Aristote. Robert le Normand est peut-être identifiable à Robert Godefroy, le traducteur en français du Liber novem judicum en 1361, qui était maître ès arts. Voir supra, note 7.

36. Sur ces différentes parties de l'astrologie, voir M. PréAud, Les Astrologues à la fin $d u$ Moyen Âge, Paris, 1984, p. 65-156, et N. WeILL-PAROT, Les «images astrologiques » au Moyen Âge et à la Renaissance. Spéculations intellectuelles et pratiques magiques, Paris, 2002.

37. Voir notamment J.-P. BoudET, « Astrologie et politique dans la crise du milieu du XIV ${ }^{e}$ siècle : le Livre des elections universelles des douze maisons de Pèlerin de Prusse », dans Par les mots et les textes. Mélanges de langue, de littérature et d'histoire des sciences médiévales offerts à Claude Thomasset, Paris, 2005, p. 83-104. 
ment par les autorités de l'Église et de l'État sur l'enseignement de la science des étoiles.

La vigoureuse campagne menée depuis les environs de 1360 par les théologiens Nicole Oresme et Henri de Langenstein (ou de Hesse) contre la divination astrale n'a donc peut-être pas été aussi inutile qu'on le pensait autrefois ${ }^{38}$. Sans doute, Charles V lui-même ne semble jamais avoir renié la passion de sa jeunesse, puisque le cahier qui contient les horoscopes de ses enfants date de $1373-1377^{39}$. Mais rien n'indique que ces horoscopes semi-officiels, dont les coordonnées étaient encore connues, en 1407-1408, d'un astrologue comme le bénédictin normand Simon de Boesmare ${ }^{40}$, aient fait l'objet, à la demande du roi, de jugements qui auraient dépassé le stade médical, personnel et familial. Et il en va de même pour l'élection astrologique qui présida à la célébration, en janvier 1377, du mariage du fils d'Amédée VI de Savoie et de Bonne, fille du duc de Berry, effectuée par Thomas de Pizan et sans doute approuvée par le roi, qui assista à la cérémonie ${ }^{41}$ : on ne sort pas du cadre familial. L'astrologie judiciaire, sous le règne de Charles V, n'a jamais été vraiment reconnue d'utilité publique. Elle est essentiellement réservée à l'usage privé du souverain. Aucun personnage, y compris Thomas de Pizan, n'apparaît encore d'une façon régulière, dans les lettres royales ou les comptes de l'Hôtel, en tant qu' «astrologien » du roi $^{42}$.

38. Sur les écrits anti-astrologiques d'Oresme, voir G. W. Coopland, Nicole Oresme and the Astrologers. A Study of his Livres de divinacions, Liverpool, 1952; S. CAROTI, "Nicole Oresme : Quaestio contra divinatores horoscopios », Archives d'Histoire Doctrinale et Littéraire du Moyen Âge, t. XLIII (1976), p. 201-310; ID., «La critica contro l'astrologia di Nicole Oresme e la sua influenza nel Medioevo e nel Rinascimento », Atti dell'Academia nazionale dei Lincei (Mem. classe di scienze morali, storiche e filologiche), ser. 8, 23 (1979), p. 545-685 ; M. LeJBowicz, «Chronologie des écrits anti-astrologiques de Nicole Oresme. Étude sur un cas de scepticisme dans la deuxième moitié du $\mathrm{XIV}^{\mathrm{e}} \mathrm{s}$. », dans Autour de Nicole Oresme, J. Quillet éd., Paris, 1990, p. 119-176. Sur Henri de Langenstein, voir H. PRUCKNER, Studien zu den astrologischen Schriften des Heinrich von Langenstein, Leipzig-Berlin, 1933. L'on remarquera que la Questio de cometa, composée par ce théologien à l'occasion du passage de la comète de 1368 dans le ciel parisien et des jugements astrologiques qu'il a suscités, est une commande de Charles V ( $c f$. Pruckner, p. 89 : «Ista questio de cometa determinata est Parisius per reverendissimum Henricum de Hassia, artium et sacre theologye monarcham magistrum, ad mandatum christianissimi regis Caroli V »). La fondation du collège de maître Gervais s'inscrit dans une perspective analogue, celle d'une incitation au développement des spéculations intellectuelles au sein de l'université de Paris, dans des limites acceptables pour la doctrine chrétienne.

39. Oxford, St John's College, ms. 164, $\mathrm{f}^{\mathrm{0}} 158 \mathrm{v}^{\mathrm{o}}-160 \mathrm{v}^{\circ}$. Sur ces horoscopes, voir E. Poulle, « Horoscopes princiers des XIV et $\mathrm{XV}^{\mathrm{e}}$ siècles », Bulletin de la Société nationale des antiquaires de France, séance du 12 février 1969, p. 63-77, notamment p. 63-69, art. réimpr. dans ID., Astronomie planétaire au Moyen Âge latin, Aldershot, 1996, texte VIII.

40. Voir J.-P. Boudet, Lire dans le ciel. La bibliothèque de Simon de Phares, astrologue $d u X^{e}$ siècle, Bruxelles, 1994, p. 124-126 et planche XXV.

41. C. de PISAn, Le Livre des fais et bonnes mours du sage roy Charles V, S. Solente éd., Paris, 1936-1940, réimpr. Genève, 1977, t. I, p. VIII.

42. Sur Thomas de Pizan, voir E. WickersheIMER, Dictionnaire, op. cit., t. II, p. 764-765 ; Christine de Pisan, Le livre des fais, op. cit., t. I, p. I-XV ; N. WeILl-PAROT, Les «Images astrologiques », op. cit., p. 605-608 et 897-900 ; J.-P. BOUDET, Entre science et «nigromance ». Astrologie, divination et magie dans l'Occident médiéval (XII ${ }^{e}-\mathrm{XV}^{e}$ s.), Paris, 2006, p. 403-408. Dans 
Certes, rien ne permet de dire que la passion personnelle de celui que Christine de Pizan qualifie de «roy astrologien » ait diminué, entre le temps où il n'était encore que dauphin et la fin de son règne. Mais il a dû composer avec le courant représenté par les adversaires de l'astrologie divinatoire, nombreux parmi ses proches. La décision de fonder deux bourses de mathématiciens au collège de Maître Gervais semble ainsi se situer dans une atmosphère analogue à celle du Songe $d u$ Vergier, largement influencée par Nicole Oresme : «La principal fin d'astrologie, si n'est mie de jugier dez choses advenir, mez sa principal fin est car elle donne cognoissance de Nostre Createur, et [...] de monstrer que la pensee humaine doit estre loings dez choses terrenes et doit plus vaquier a contemplacion ${ }^{43}$. »

Gervais Chrétien était-il d'ailleurs lui-même un praticien de l'astrologie? C'est ce que prétend Simon de Phares en lui attribuant d'avoir prédit la date de la mort de Jean le Bon, en 1364. Mais il n'est renseigné sur cet événement que par ses sources historiographiques, la Chronique de Jean II et Charles V et le Compendium de Robert Gaguin ${ }^{44}$, et non par un quelconque jugement astrologique émanant de la plume de Gervais Chrétien. Des écrits de ce dernier, il ne reste malheureusement qu'un bref opuscule contre la peste, où il n'est pas question d'astrologie ${ }^{45}$. Le seul témoignage dont on pourrait disposer pour apprécier l'intérêt personnel du médecin de Charles V pour la science des astres réside dans le fait qu'à la suite du superbe exemplaire du Livre de Jehan de Mandeville qu'il a fait copier et donné au roi, en $1371^{46}$, se trouve la traduction française d'une version du De preservatione ab epidimia

une donation du 21 avril 1372, Charles V l'appelle «nostre astronomien». Mais il est par ailleurs exclusivement désigné par le titre de «physicien». C'est une différence majeure avec les astrologues de cour de la seconde moitié du $\mathrm{Xv}^{\mathrm{e}}$ siècle.

43. Le Songe du Vergier, éd. d'après le manuscrit Royal 19 C IV de la British Library, par M. Schnerb-Lievre, Paris, 1982, t. I, p. 408 (dernière réplique du Chevalier sur la question). Voir J. Quillet, La Philosophie politique du Songe du Vergier (1378). Sources doctrinales, Paris, 1977, p. 105-122.

44. La mort de Jean II est relatée dans Les Grandes Chroniques de France. Chronique des règnes de Jean II et de Charles V, R. Delachenal éd., Paris, 1910-1920, vol. I, p. 341, et par R. GaGuin, Compendium de origine et gestis Francorum, Paris, P. Le Dru, 1495, VII, fo 74.

45. Ce traité aujourd'hui perdu a été édité par P. d'Hermansart, « Une ordonnance médicale contre la peste vers 1400 », Bulletin de la Société des antiquaires de la Morinie, 10 (1900), p. 617622. Il présente plusieurs passages communs avec une «collation faite à Paris, approuvié par les maistres en medecine pour l'espidimie », conservée dans deux manuscrits : voir D. JACQUART, La Médecine médiévale, op. cit., p. 258-260.

46. Paris, BnF, ms. nouv. acq. fr. 4515, copié par Raoulet d'Orléans en 1371. Voir L. Delisle, Recherches, op. cit., t. I, p. 275-276 ; La Librairie de Charles V, Paris, 1968, p. $113-$ 114 et pl. 19 ; R. H. Rouse et M. A. Rouse, Illiterati et uxorati. Manuscripts and their Makers. Commercial Book Producers in Medieval Paris, 1200-1500, Turnhout, 2000, vol. I, p. 273-279 (sur Raoulet d'Orlans). Le deuxième médaillon du frontispice du fo 1 de ce ms. montre Gervais Chrétien offrant le Livre de Mandeville au roi. Ce volume constitue le plus ancien exemplaire de la version continentale de cet ouvrage, publiée par M. LETTs, Mandeville's Travels, Londres, 1953, 2 vol. : voir C. Deluz, Le Livre de Jehan de Mandeville, une "géographie » au XIVe siècle, Louvain-la-Neuve, 1988. 
de Jean de Bourgogne, dit à la Barbe ${ }^{47}$, traduction dont Gervais a toutes les chances d'être le commanditaire, sinon l'auteur, et qui se montre résolument favorable à une interprétation astrologique des épidémies de peste et des autres catastrophes qui se sont abattues sur l'Europe au XIV siècle :

Pour ce que toutes choses tant element comme elementees sont gouvernees de leur plus haut, si comme dit Mesehalac ou Livre des interpretacions $^{48}$, les choses creees en souveraineté, toutes les autres qui sont dessoubz leurs cercles, essence, nature, substance, croissement et deffault, donnent et administrent, dont il advient que par l'influence des corps supercelestiaux, causeement et de fait, li airs soit de pieçea corrumpus et effect pestilentieux apparus. Mais n'est mie a entendre que airs soit corrumpus en sa substance, car c'est un corps simples, mais par les causes des males vapeurs receues et entremellees est corrumpus, dont epidimie en plusieurs lieux est ensuivie ${ }^{49}$.

Suit un passage attendu, où l'auteur reprend le topos pseudo-hippocratique en posant la question: "De quoy se mesle phisiciens ignorant astrologie ? Nulz ne se doit mectre en ses mains, car li habituacions de astrologie et de art de medicine rieulent li uns l'autre, et moult suppose l'une science de l'autre, car chascune en chascun ne se peut desclairier, et moult de fois l'ai trouvé par experience en pratiquant par l'espace de XL ans et plus ${ }^{50}$. »

Jean de Bourgogne rappelle ensuite que les causes célestes étant les causes premières, il convient de les connaître, car « la cause souveraine ignoree, la cognoissance des derraines est ostee, [et] comme la cause premiere plus influe en son effect que la cause seconde, comme il est dit ou premier [cha-

47. Sur ce personnage énigmatique, dont l'opuscule semble avoir été composé à Liège, en 1366 (et non pas en 1365, comme il est dit le plus souvent), voir E. Wickersheimer, Dictionnaire, op. cit., t. I, p. 369-370, et Supplément par D. JACQUART, p. 147-148. Le texte latin du De preservatione présente de notables différences entre une version purement médicale et une version astrologico-médicale : voir G. GutTMann, Die Pestschrift des Jean à la Barbe, Berlin, 1903, p. 10-18 ; K. SudHOFF, «Pestschriften aus den ersten 150 Jahren nach der Epidemie des "schwarzen Todes" 1348, III », Archiv für Geschichte der Medizin, vol. V (1912), p. 58-69. La traduction française suit l'une des branches de la seconde version : voir l'éd. fautive qu'en donne D. W. Singer, "Some Plague Tractates (Fourteenth and Fifteenth Centuries) », Proceedings of the Royal Society of Medicine, Section of the History of Medicine, t. IX-2 (1915-1916), p. 200212, et infra.

48. Référence (qui ne se trouve pas dans l'original latin) au Libellus de intentionibus secretorum astronomie, ou De cogitationibus ab intentione de l'astrologue juif Messahala : voir F. J. CARmody, Arabic Astronomical and Astrological Sciences, op. cit., p. 28-29, et L. THORNDIKE, « The Latin translations of astrological works by Messahala », Osiris, 12 (1956), p. 49-72, aux p. 56-62.

49. D. W. Singer, «Some Plague Tractates », loc. cit., p. 201-202, collationné avec le ms. Paris, BnF, nouv. acq. fr. $4516, \mathrm{f}^{\mathrm{0}} 97$.

50. Ibid., p. $202 ; \mathrm{ms}$. cité, $\mathrm{f}^{\circ} 97 \mathrm{v}^{\circ}$. Sur la littérature astrologico-médicale pseudo-hippocratique, voir notamment L. THORNDIKE, « The three Latin translations of the pseudo-hippocratic tract on astrological medicine », Janus, t. 49 (1960), p. 116-129 ; P. KIBRE, " "Astronomia" or "Astrologia Ypocratis" ", dans Science and History: Studies in Honor of Edward Rosen, WorclawVarsovie, 1978 (Studia Copernicana, XVI), réimpr. dans EAD., Studies in Medieval Science. Alchemy, Astrology, Mathematics and Medicine, Londres, 1984, p. 33-56. 
pitre] Des causes $^{51}$, ainsi appert que senz astrologie, en ce cas cy especial, proffite peu phisique, pour quoy par deffaute de conseil moult de genz perissent $»^{52}$. Et l'auteur achève son opuscule par une interprétation astrologique des effets présents et futurs des conjonctions de Saturne et Jupiter de 1345 et 1365 :

Item, ne croie nulz que ceste pestilence [celle de 1365-66] soit pour la raison de Saturne et Jupiter, pour la conjunction qui se fist d'eulz et d'autres l'an passé, c'est assavoir en l'an LXV, ainçois vient des reliques de l'autre conjunction autreffoiz faite de l'an XLV, dont les traces apperent encore en effect. L'autre conjunction fist son influence et pestilence tant seulement en et sur corps humain, mais aussi en besteaulx cherra la pestilence et moult d'autres maulx par succession de temps seront semé en monde, comme grans guerres, mutacions de royaumes et de sectes, et destructions d'autres sectes, paours, angoisses, riotes et discordes, famines, mortalitez, depauparacions de richesce de moult, et occisions de puissans hommes, destruction de sectes de Sarrasins, et moult de pluyes et en pluseurs lieux grans deluges, deffaut de fruiz, et moult de perilz a ceuls qui passeront la mer, et moult de signes et espoventauz apperont en l'air, et seront moult de tonnoires et de foudres et de soufflemens de vens fors et vertueux et par tres grans tempestes, et sera en l'Eglise tribulacion grande et discorde, et oppression d'aucuns grans prelas et de ministres de l'Eglyse, et moult d'autres inoppinees merveilleuses et esbahissans, par succession de temps, dont en singulier seroit lonc a raconter, et non mie tost ces choses avenront, mais passé trois ans de ceste nouvelle conjunction a compter, les grans merveilles plus que devant apperont et commenceront a pululer, c'est assavoir destrempance d'air, froidures, pluyes et grans diminucions d'yaues, et aprés grans deluges et inundacions d'eaues, et deffaux de fruis et moult d'autres en ces trois annees apperront. Mais les grans advenemens se tarderont encore de venir. De ces et en autres adversitez nous vueille Dieu gouverner. Amen ${ }^{53}$.

Voilà qui dépasse de loin le cadre de l'astrologie médicale et qui se rapproche des prédictions astrologico-prophétiques sur les conjonctions de 1365 et 1385 , dont celle du juriste bolonais Giovanni da Legnano constitue l'un des meilleurs exemples ${ }^{54}$. Or c'est trois pages plus loin, au folio $102 \mathrm{v}^{\mathrm{o}}$, que se trouve l'ex-libris effacé du destinataire du codex : «Ce livre est a nous, Charles le $\mathrm{V}^{\mathrm{e}}$ de nostre nom, roy de France, et le nous donna metre

51. Référence au Liber de causis pseudo-aristotélicien : «Toute cause première influe plus sur son effet que la cause universelle seconde », dans P. MAGNARD et al., La Demeure de l'être. Autour d'un anonyme. Étude et traduction du Liber de causis, Paris, 1990, p. 41.

52. D. W. Singer, «Some Plague Tractates», loc. cit., p. 204 ; Paris, BnF, ms. nouv. acq. fr. $4516, \mathrm{f}^{\circ} 97 \mathrm{v}^{\circ}$.

53. Ibid., p. 209 ; ms. cité, fo $100 \mathrm{v}^{\circ}-101$.

54. La version latine de ce texte est conservée sans nom d'auteur dans le ms. Paris, BnF, lat. 2599, $\mathrm{f}^{\mathrm{o}} 267 \mathrm{v}^{\circ}-270$, alors que sa version italienne, étudiée et publiée par E. GiANAZZA, La vita e le opere di Giovanni da Legnano (sec. XIV), Parabiago, 1973, p. 258-275, est explicitement attribuée à Legnano. 
Gervese Cretien, notre premier fisicien, l'an M.CCC.LXXI. » Et il est difficile de ne pas voir dans cette mention de possession, que Charles $\mathrm{V}$ réservait aux livres qu'il chérissait le plus, une marque ostensible d'approbation envers la forme et le contenu de l'ensemble du volume ${ }^{55}$.

\section{Les manuscrits et activités scientifiques de Gervais Chrétien, du collège et de ses boursiers : quelques données}

Les autres livres provenant de Gervais Chrétien et mentionnés dans les inventaires de la librairie du Louvre nous ramènent à sa fonction de premier médecin du roi, puisqu'il s'agit de deux exemplaires du Canon d'Avicenne. Le premier, donné à Charles V avant 1373, est décrit comme suit : «Un livre d'Avicene complet, a IIII fermoirs d'argent et couvert de soie blanche, et le donna au roy [Charles V] maistre Gervaise Chrestien, son premier phisicien », une note postérieure ayant ajouté que «le roy [Charles VI] l'a fait bailler a maistre Regnaut Freron », sans doute lorsque ce dernier fut nommé premier médecin de Charles VI, en $1387^{56}$. Le second, entré dans la librairie royale avant 1411, prisé 15 livres parisis en 1424, était quant à lui « un beau livre de medecine qui se appelle Canones Abohaaly, escript de bonne menue lettre de forme, en latin, a deux coulombes, en assez gros volume. Comm. : Cap. XXVIII de causis. Fin : eperzagel, id est perditis. Et fu ledit livre maistre Gervaise Christien, comme il est escript en la fin dudit livre; couvert de cuir a empreintes, sans ais, fermant a IIII lasnieres ${ }^{57}$.

Or on retrouve cette place privilégiée accordée au Canon d'Avicenne dans le testament de Gervais Chrétien, daté du 15 avril 1382, où la quatrième clause stipule qu'il lègue « aux écoliers de Sainte-Marie du diocèse de Bayeux, établis dans ses maisons à Paris, tous ses livres de médecine, sauf son Avicenne en deux petits volumes, qui seront vendus pour employer leur prix par ses exécuteurs à des usages pieux ${ }^{58}$. Et Karine Rebmeister-Klein a identifié

55. Rappelons que les mss. nouv. acq. fr. 4515 et 4516 ne formaient qu'un seul volume à l'origine. Sur les livres sur lesquels Charles V a apposé sa signature, voir L. DeLISLE, Recherches, op. cit., t. I, p. 3-5, et J.-P. BoudET, «Le bel automne de la culture médiévale, XIV'-XV ${ }^{\mathrm{e}}$ siècles », dans Histoire culturelle de la France, J.-P. Rioux et J.-Fr. Sirinelli dir., t. I, Le Moyen Âge, M. Sot dir., Paris, 1997, p. 280-281.

56. L. Deliste, Recherches, op. cit., t. II, p. 133-134. Sur Regnault Freron, qui avait été boursier du collège de Navarre, voir E. WICKERSHEIMER, Dictionnaire, op. cit., t. II, p. 689-690, et Supplément par D. JACQUART, p. 255 ; N. GoROCHOv, Le Collège de Navarre de sa fondation (1305) au début du XV siècle (1418). Histoire de l'institution, de sa vie intellectuelle et de son recrutement, Paris, 1997, p. 635-637 ; B. GuENÉE, La Folie de Charles VI, op. cit., p. 108-109, $111,113-116,118,121,123-128$.

57. L. DelisLe, Recherches, op. cit., t. II, p. 133.

58. S. Le Paulmier, « Notice sur Gervais Chrétien», loc. cit., p. 119 : «Item, lego scolaribus beate Marie de diocesis Bajocensis, in domibus meis Parisius fundatis, omnes libros meos medicine, excepto libro tamen Avicenne in duobus parvis voluminibus, quem volo vendi et piis usibus per executores meos converti. » 
deux codices subsistants provenant de cette donation. Il s'agit de deux manuscrits conservés à la Bibliothèque de la Sorbonne : l'un, acheté 16 écus d'or par Gervais Chrétien à la veuve et aux exécuteurs testamentaires du libraire parisien Mathieu Vavasseur, en 1352, contient le texte complet du Canon d'Avicenne dans la traduction de Gérard de Crémone ${ }^{59}$; dans l'autre, copié à Paris, en 1377, à la demande de Maître Gervais, se trouve le commentaire de Dino del Garbo sur la $4^{\mathrm{e}}$ fen du livre I du même Canon ${ }^{60}$.

Il n'existe malheureusement aucun catalogue des livres du collège de Maître Gervais datant de la période médiévale, mais l'on sait qu'il était prévu qu'ils soient répartis dans deux bâtiments distincts, celui des théologiens et celui des artiens : le chapitre VIII des statuts de 1378 évoque l'existence de deux bibliothèques différentes avec des livres enchaînés pour l'étude, logés dans ces deux maisons : dans la libraria theologorum erunt libri ad theologos et medicos pertinentes, et libri logicales et philosophie et mathematicales in libraria artistarum ${ }^{61}$. Les livres de médecine devaient donc se trouver dans la libraria theologorum et les livres de quadrivium dans la libraria artistarum, ce qui ne facilitait guère, a priori, les velléités d'interdépendance entre la médecine et l'astronomie-astrologie. Ce chapitre des statuts n'a peutêtre pas été toujours appliqué à la lettre, mais les donations de livres effectuées par Guillaume Vauchis (1414) et par Jean Blondel (1474) en faveur du collège, ainsi que les inventaires des deux librarie, perdus mais mentionnés dans le répertoire des documents possédés par le collège en 1472, s'inscrivent clairement dans cette bipartition originelle ${ }^{62}$.

Cette structure, parvenue à maturité au début du règne de Charles VI, semble, en effet, avoir perduré au $\mathrm{XV}^{\mathrm{e}}$ siècle. Dans le seul compte conservé

59. Paris, Bibl. de l'Univ., ms. 130, dont voici le colophon du fo 369 : «Iste liber est Gervasii Christiani, magistri in artibus et medicina Parisius, et emit eum ab uxore et executoribus Mathei Vavassoris deffuncti, quondam librarii in vico novo Beate Marie commorantis, anno Domini millesimo $\mathrm{CCC}^{\circ}$ quinquagesimo secundi, die mercurii vicesima nona augusti, et constitit sexdecim bona scuta auri. » Notice dans K. RebmeIster-Klein, Les Livres des petits collèges, op. cit., t. III, p. 1099-1100.

60. Ibid., t. III, p. 1101-1102. Il s'agit du ms. Paris, Bibl. de l'Univ. 134, dont voici le colophon, au fo 260 : «Iste liber est Gervasii Christiani, regis Francie medici, qui fecit eum scribi anno Domini $1377^{\circ}$, Parisius. » Sur le médecin italien Dino del Garbo et son commentaire du Canon, dont la partie en question a été composée entre 1311 et 1318, voir E. WiCKERSHEIMER, Dictionnaire, op. cit., t. I, p. 119-120, et Supplément par D. JACQUART, p. 60-61 ; N. G. SIRAISI, Taddeo Alderotti and his Pupils. Two generations of Italian medical learning, Princeton, 1981, p. 55-64.

61. P. de Longuemare, «Le collège de maître Gervais Chrétien », loc. cit., p. 291.

62. K. ReBMEISTER-KLein, Les Livres des petits collèges, op. cit., t. II, p. 693-696. En 1414, Guillaume Vauchis donne 12 volumes de théologie, de philosophie et d'histoire à la libraria theologorum, et 6 volumes de philosophie à la libraria artistarum. Tous ces livres devront être enchaînés. Paris, Bibl. de l'Univ., ms. 969 (inventaire et répertoire des lettres appartenant au collège, faits par Raoul de Montfiquet en 1472, et continués par Étienne Pothier, procureur du collège, en 1525), fo $42-42 \mathrm{v}^{0}$ : «IIII'XVII. Item, inventarium librarie artistarum collegii magistri Gervasii Christiani in uno rotulo pergameni. IIII'XVIII. Item, inventarium libraire [sic] theologorum collegii magistri Gervasii in quodam rotulo pergameni. » 
du collège pour la période médiévale, qui concerne l'année 1409, on trouve des mentions relatives à plusieurs lecture données en son sein, à deux bibliothécaires distincts, à la fabrication et à la copie d'un exemplaire des Moralia in Job de saint Grégoire, à l'achat d'un livre de médecine et des Quodlibeta d'Henri de Gand, à l'activité de maître Henri de Fontaines, un boursier en théologie considéré par Guillebert De Mets comme «l'astrologien » par excellence du Tout-Paris de l'époque ${ }^{63}$, et au transport d'une sphère [armillaire ?] du domicile de « Furoris », alias Jean Fusoris, au collège ${ }^{64}$. Voilà qui semble confirmer l'hypothèse selon laquelle une bonne partie des livres qui n'étaient pas donnés en legs au collège de Maître Gervais étaient achetés à l'extérieur mais que cet établissement bénéficiait peut-être aussi, au moins d'une manière occasionnelle, du travail d'un scriptorium interne, une dizaine de manuscrits subsistants y ayant été copiés par un petit nombre de scribes ${ }^{65}$. Quant à la mention de Fusoris, elle donne quelque consistance au témoignage de Simon de Phares sur la présence de «speres, astrolabes, saphee et autres instrumens servans a ladicte science » dans la « librairie » du collège ${ }^{66}$, et elle ouvre des perspectives sur la circulation des savoirs scientifiques dans la capitale du royaume de France au début du $\mathrm{Xv}^{\mathrm{e}}$ siècle : maître en médecine et bachelier en théologie, Jean Fusoris était le plus grand constructeur d'instruments

63. Guillebert De Mets, Description de la ville de Paris, dans Le Paris de Charles $V$ et de Charles VI vu par des écrivains contemporains, Le Roux de LinCY et L. M. TISSERAND éd., Paris, 1867, réimpr. Caen, 1992, p. 163 (p. 233 de l'éd. originale). Sur Henri de Fontaines, clerc du diocèse de Bayeux, maître ès arts et bachelier en théologie, voir Chartularium Universitatis Parisiensis, op. cit., t. IV, Paris, 1897, p. 2, no 1715 ; p. 148-150, n 1843-1845, et p. 206, $\mathrm{n}^{\circ} 1918 \mathrm{n}$; H. Millet et E. Poulle, Le Vote de la soustraction d'obédience en 1398, t. I, Introduction, édition et fac-similés des bulletins de vote, Paris, 1988, p. 192-193, nº 164 ; Arch. nat., M 167, pièce $\mathrm{n}^{\circ} 17$, fondation de deux bourses par Guillaume de Lirois, en mai 1402 (liste des membres du collège à cette date).

64. Paris, Arch. nat., M 165, pièce $\mathrm{n}^{\circ} 23$, compte sur rouleau tenu par Jean Manchon, maître ès arts et prêtre du diocèse de Bayeux (Chartularium, op. cit., t. IV, p. 97, no 9), dans la rubrique Misie extraordinarie: «Item, $\mathrm{X}^{\mathrm{a}}$ maii [1409], pro obitu fondatoris nostri. Item, eodem die, pro lectura magistri Michaelis Le Voydre [cité plus haut comme prieur]. Item, pro lectura mea [...] Item, una bota vitulini pro Moralibus Gregorii. [...] Item, pro apportando speram de domo Furoris et falcando partum theologorum. [...] Item, pro lectura mea VIII ${ }^{\mathrm{a}}$ junii. Item, $\mathrm{XXI}^{\mathrm{a}}$ junii, pro uno libro medicine noviter empto. [...] Item, pro Quolibetis de Gandavo noviter emptis. [...] Item, pro pergameno proponendo in duobus voluminibus Moralium Gregorii. Item, pro colando alterius dictorum voluminum. Item, pro religando ambo volumina dictorum Moralium. [...] Item, pro scriptura hujus quod deficiebat in dictis Moralibus. » Plus bas, dans la rubrique Arreragia collegio debita : « M. Henricus de Fontanis, pro camera, etc., et pro mappis. Item, ipsemet pro panno quod sibi vendidit collegium. [...] M. Guillemus de Estervilla pro libraria. Henricus de Louvigny pro eadem causa. »

65. K. ReBMeISTER-KLein, Les Livres des petits collèges, op. cit., t. I, p. 197-199, et t. III, p. 1092-1093. C'est le cas, en particulier, des mss. 183, 184 et 191-192 de la Bibl. de l'Univ., copiés par Jean Picot dans le dernier quart du $\mathrm{Xv}^{\mathrm{e}}$ siècle.

66. Rappelons que le collège de Sorbonne abritait lui aussi des instruments de cette sorte qui étaient régulièrement empruntés par ses boursiers au $\mathrm{XV}^{\mathrm{e}}$ et au début du $\mathrm{XVI}^{\mathrm{e}}$ siècle : voir Le Registre de prêt de la bibliothèque du collège de Sorbonne, 1402-1536, J. VIEILLARD éd., Paris, 2000 (Documents, études et répertoires publiés par l'IRHT, 57), p. 789-790. 
astronomiques de son temps ${ }^{67}$ et l'on sait qu'il hébergeait, vers 1410, dans une demeure parisienne et en compagnie de Jean de Châlon, médecin de Jean sans Peur, des «écoliers » à qui il donnait peut-être des cours d'astronomie ${ }^{68}$.

La guerre civile et la crise politique des années 1411-1436 ont certainement affecté l'établissement fondé par Gervais Chrétien, même s'il n'a pas subi le même sort que le collège de Navarre en 1418 et si les Anglais ont ménagé les collèges normands. En tout cas, ce n'est que dans le dernier tiers $\mathrm{du} \mathrm{XV}^{\mathrm{e}}$ siècle que la documentation permet de repérer de nouveau le cursus de certains de ses grands boursiers, qui ne sont plus forcément d'origine normande. En 1472, un inventaire des lettres du collège nous révèle que Denis Le Herpeur (ou Cytharède l'aîné), du diocèse de Bayeux, docteur en théologie, a été promu à la chancellerie de l'Université ${ }^{69}$, que l'un des boursiers en médecine, Jean Michel, un Angevin, a passé son baccalauréat ${ }^{70}$, alors que trois autres étudiants résidant au collège ont été reçus à la licence en médecine : il s'agit du bursarius astrologus Hervé Avis - le frère de Jean Avis, lui-même doyen de la faculté de médecine parisienne et futur ami de Simon de Phares ${ }^{71}$-, qui se fit Célestin juste après avoir passé sa licence, comme le dit 1'auteur du Recueil ${ }^{72}$, et de deux étudiants promus au doctorat: le boursier en médecine Guillaume Miette, alors procureur de la nation

67. Voir E. Poulle, Un constructeur d'instruments astronomiques au XV siècle. Jean Fusoris, Paris, 1963, et en dernier lieu F. CASI, «A Medieval Astrolabe in the Tradition of Jean Fusoris », Nuncius. Annali di storia della scienza, XIX-1 (2004), p. 3-29, qui recense 31 astrolabes subsistants sortis de l'atelier de Fusoris.

68. Voir L. Mirot, «Le procès de maître Jean Fusoris, chanoine de Notre-Dame de Paris (1415-1416) », Mémoires de la Société de l'Histoire de Paris et de l'Ile-de-France, t. XXVII (1900), p. 137-237, aux p. 144 et 235, où il est question de la situation difficile créée par la mort de Jean de Châlon pour le «regimen sue domus, ac pluritatem scolarium existencium ». Sur Jean de Chalon, qui fut «physicien » des ducs de Bourgogne Philippe le Hardi et Jean sans Peur et chanoine de la Sainte-Chapelle de Dijon, voir E. WiCKERSHEIMER, Dictionnaire, op. cit., t. I, p. 380, et Supplément par D. JACQUART, p. 150.

69. Paris, Bibl. de l'Univ., ms. 969, fo 2-2 $\mathrm{v}^{\circ}$. Sur Denis Le Herpeur ou Cytharède l'aîné $(\dagger$ 1482), voir Du Boulay, Historia, op. cit., t. V, p. 871.

70. Sur Jean Michel, bachelier en médecine à Paris en 1472, licencié en 1476, maître régent de la faculté de médecine d'Angers en 1484, engagé comme médecin de la ville d'Angers en 1486, année où il fit représenter dans cette ville un remaniement du Mystère de la Passion d'Arnoul Gréban, et qui devint l'un des médecins de Charles VIII et de son fils, Charles-Orland, voir infra et note 79.

71. Sur Jean Avis l'aîné († 1501), doyen de la faculté de médecine de Paris à partir de 1470, qualifié de « conseiller et médecin du roi » en 1492, qui devint l'ami de Simon de Phares à cette époque, posséda plusieurs manuscrits astronomiques et astrologiques, et prêta l'un d'entre eux, du plus haut intérêt, à l'auteur du Recueil, voir E. WICKERSHEIMER, Dictionnaire, op. cit., t. I, p. 356, et Supplément par D. JACQUART, p. 143 ; J.-P. BoudeT, Lire dans le ciel, op. cit., p. 175-189, et Le Recueil, op. cit., t. I, p. 595-596.

72. Sur Hervé Avis, voir E. WicKersheIMER, Dictionnaire, op. cit., t. I, p. 295, et Le Recueil, op. cit., t. I, p. 601. Hervé Avis fut le possesseur du ms. Paris, BnF, lat. 7407, qui comprend plusieurs traités astronomiques de Jean de Lignères et de Jean de Saxe, mais aussi, au f ${ }^{\circ} 69$, un horoscope de la nouvelle lune du 13 mars 1469 et des notes sur l'influence de la Lune lors de la conception, qui semblent de sa main. 
normande de l'Université, et Gilbert Droulin, lui aussi angevin d'origine, qualifié d'hospes in dicto collegio, l'accueil d'hôtes au sein du collège étant effectivement prévu par les statuts de $1378^{73}$. En 1484, une autre relation de Simon de Phares, Geoffroy du Saussay, apparaît comme boursier et licencié en médecine dans un acte d'arbitrage de Geoffroy de Pompadour, grand aumônier de France, évêque de Périgueux et proviseur du collège, au sujet d'un conflit entre dix grands boursiers résidant dans la domus theologorum et deux maîtres ès arts, Alain Pothier et Robert de Orto, qui en avaient visiblement été chassés par leurs confrères ${ }^{74}$. J'ai même retrouvé la liste des douze grands boursiers du collège de Maître Gervais en 1492 et constaté que les deux astrologi, Denis Cytharède (le jeune) et Pierre Rosée, connus par ailleurs pour être étudiants en médecine, le premier en 1476-1480 et le second depuis 1487 , ne sont pas mentionnés dans le Recueil ${ }^{75}$. De surcroît, Pierre Rosée devint procureur et receveur général du collège en 1494-1495 ${ }^{76}$. Le fait que Simon de Phares soit resté silencieux à leur égard alors qu'il les connaissait sûrement - il résidait à Paris à cette époque et il eut accès à l'une des bibliothèques du collège, soit par la faveur de Charles VIII, soit avec la complicité de l'un des boursiers qui en avait la clef - est significatif.

73. Paris, Bibl. de l'Univ., ms. 969, fo $2 \mathrm{v}^{0}$ : « Eodem insuper anno [1472], fuerunt tres licentiati in medicina ejusdem collegii scolares, scilicet magister Herveus Avis, bursarius astrologus, qui eodem anno factus est celestiniensis, et predictus Guillemus Miecte et magister Gilbertus Droulin, hospes in dicto collegio, qui duo stanti doctores in medicina effecti sunt. » Sur Guillaume Miette et Gilbert Droulin, voir E. WicKerSheIMER, Dictionnaire, op. cit., t. I, p. 193 et 256, et Supplément par D. JACQUART, p. 108. Gilbert Droulin fut nommé médecin de la ville d'Angers en 1486, comme Jean Michel. Guillaume Miette devint, à l'instar de ce dernier, médecin de Charles VIII, et fut envoyé en sa compagnie à Angers en 1486 pour s'enquérir «s'il y avait quelque danger de mortalité ». Le collège de Maître Gervais a certainement été utile à la constitution de ce réseau de médecins angevins bien en cour. Les chapitres XII et XIV des statuts de 1378 traitent de l'accueil des hôtes : voir P. de Longuemare, « Le collège de maitre Gervais », loc. cit., p. 300-302 et 305-306.

74. Arch. de $1^{\prime}$ Univ. de Paris, carton $n^{\circ} 21$, liasse 1 , pièce $n^{\circ} 1$ : «Sentencia super habitudines separatim habenda inter theologos et artistas in collegii magistri Gervasii », 17 sept. 1484. Sur Geoffroy du Saussay, qui fut maître régent de la faculté de médecine de Paris, de 1485 à sa mort, en 1525, voir E. WickersheIMER, Dictionnaire, op. cit., t. I, p. 178 ; Le Recueil, op. cit., t. I., p. 595-596. Sur Geoffroy de Pompadour, $c f$. X. de LA SElle, Le Service des âmes à la cour, op. cit., p. 304-305.

75. Arch. de $1^{\prime}$ Univ. de Paris, carton $n^{\circ} 21$, liasse 1 , pièce $n^{\circ} 5, f^{\circ} 3-3 v^{\circ}, 18$ juillet 1492 : «Stephanus Grandes, Robertus de Orto, Guilelmus Bachelier, Guillermus de Quercy, Petrus Barvalles, Dionisius le Masuyer cum predicto Auray, theologi; Robertus Vaugelin, Ricardus Gassion, medici ; Dyonisius Cytharedi, Petrus Roris, astrologi ; Johannes du Moustier, decretista... » Sur Denis Cytharède (ou Le Herpeur) le jeune, homonyme et parent du précédent, et Pierre Rosée, voir E. WickersheImer, Dictionnaire, op. cit., t. I, p. 115, et t. II, p. 659. Sur les deux boursiers medici, l'un, Richard Gassion, se vit interdire de prendre part à l'élection du recteur de l'université de Paris en 1492 mais fit ensuite une belle carrière comme doyen de la Faculté en 1502-1504 et médecin de Charlotte d'Armagnac, épouse du duc de Guise ; l'autre, Robert Vaugelin (alias Vauquelin), fut reçu bachelier en médecine en 1496, licencié et maitre en 1498, mais semble avoir quitté Paris après sa réception à la maîtrise : ibid., t. II, p. 700 et 718, et Supplément, p. 258.

76. Arch. nat., $\mathrm{H}^{3} * 2875^{1}, \mathrm{f}^{\mathrm{o}} 2 \mathrm{v}^{\mathrm{o}}$. 
L'activité scientifique de ces boursiers en médecine et en astrologia des années 1470-1500 parait, en effet, avoir eu un faible rayonnement. Aucun d'entre eux n'a composé un ouvrage relevant de ces disciplines et conservé à l'heure actuelle. Quant à leurs lectures, nous n'en avons gardé que des traces très partielles. Au total, Karine Rebmeister-Klein a identifié une cinquantaine de volumes subsistants provenant du collège de Maître Gervais, dont au moins $35 \mathrm{~s}^{\prime} \mathrm{y}$ trouvaient dès le $\mathrm{Xv}^{\mathrm{e}}$ siècle ${ }^{77}$. Or la ventilation par matière de ces volumes est la suivante : théologie : 18 ; sermons, édification et autres textes religieux : 8 ; histoire : 2 ; droit : 4 , dont 3 de droit canon ; philosophie : 4 ; classiques latins : 1 ; humanisme : 1 ; médecine : 4 ; quadrivium : 5. Même si ces chiffres sont à manier avec prudence et pourraient sans doute être revus à la hausse, leur valeur relative indique que la place des sciences dans les deux bibliothèques du collège de Maître Gervais semble avoir été assez modeste, en proportion des quatre bourses sur 24 qu'y détenaient les deux médecins et les deux scholares regis.

Parmi les livres de médecine, outre les deux volumes décrits plus haut, qui provenaient de l'héritage personnel de Gervais Chrétien, ne subsistent qu'un fragment du Colliget d'Averroès (ms. Paris, Bibl. de l'Arsenal, 707 C) et un exemplaire de la $4^{\mathrm{e}} \mathrm{fen}$ de la première partie du Canon d'Avicenne, commentée par Jacques Despars et copiée par Denis Cytharède (le jeune), en $1478^{78}$. Quant aux livres de quadrivium, il s'agit, d'une part, de deux volumes légués au collège, en 1498, par Jean Michel, qui était devenu médecin de Charles VIII et de son fils, Charles-Orland ${ }^{79}-$ le ms. 593 de la Bibliothèque de l'Université de Paris, qui contient le Quadripartitum de Ptolémée avec le commentaire d'Hali Abenrudian, et le ms. BnF, lat. 7482, qui conserve deux traités d'astro-météorologie, le De mutatione temporis d'Alkindi ${ }^{80}$ et le

77. K. Rebmeister-Klein, Les Livres des petits collèges, op. cit., t. III, p. 1087-1093.

78. Paris, Bibl. de l'Univ., ms. 589, dont voici le colophon, au fo $266 \mathrm{v}^{\circ} \mathrm{b}$ : «Explicit $4^{\text {ta }}$ fen primi Canonis Avicenne cum commento ipsius, quam complevi anno Domini $1478^{\circ}$, prima die junii, fuit gracie humiles et laus Altissimo. Derpeur. Amen. » $A u f^{\circ} 269 v^{\circ}$, d'une autre main que celle du scribe, on lit ces vers : «Ceste quarte est a mon compere./ Dieu vous doint bonjour de jouy./ Il est vroy je luy veut faire,/ Parquoy j'en suys tout resjouy./ Vrayment je l'ay veu et ouy/ Tant au lundi qu'au samedi/ En l'escripvant presqu'enfouy,/ Maistre Denis Cytharedi,/ Depuys mynuyt jusque a midy. » La reliure de ce ms., qui date du XIX ${ }^{\mathrm{e}}$ siècle, mentionne, sans doute par erreur, qu'il provient du collège du Trésorier.

79. Ces deux volumes sont légués ex dono regio au collège, le 17 juillet 1498, quelques mois après la mort de Charles VIII (voir K. ReBMEISTER-KLeIN, Les Livres des petits collèges, op. cit., t. III, p. 1094). Il ne faut confondre ce Jean Michel, médecin angevin, ni avec Jean Michel de Pierrevive, un autre médecin de Charles VIII mort en 1495, ni avec un homonyme, sans doute frère mendiant, auteur d'une prophétie destinée à Charles VIII : voir E. WICKERSHEIMER, Dictionnaire, op. cit., t. II, p. 448-449; Le Mystère de la Passion: Angers, 1486, de Jean Michel, O. Jodogne éd., Gembloux, 1959, p. VII-IX ; C. Beaune, «Visionnaire ou politique ? Jean Michel, serviteur de Charles VIII », Journal des Savants, 1987, p. 65-78.

80. Voir G. Bos et C. BuRnetT, Scientific Weather Forecasting in the Middle Ages : the Writings of Al-Kindi. Studies, Editions and Translations, Londres-New York, 2000. 
Repertorium pronosticum de mutatione aeris de Firmin de Beauval ${ }^{81}$ - ; et il s'agit, d'autre part, de trois volumes mentionnés dans un fragment de catalogue de la bibliothèque du collège au XVII ${ }^{\mathrm{e}}$ siècle, dont on ignore à quelle date ils y sont entrés ${ }^{82}$ : un exemplaire de la Cosmographia de Ptolémée (ms. 981 de la Bibliothèque de l'Arsenal) et deux classiques de l'astrologie latine, le Liber introductorius ad judicia stellarum de Guido Bonatti (ms. Paris, Bibl. de l'Univ. 594) - le seul texte difficilement tolérable par l'Église ${ }^{83}$ et la Summa judicialis de accidentibus mundi de Jean d'Eshenden (ms. Paris, Bibl. de l'Univ. 598 ${ }^{84}$. Des ouvrages de référence, donc, mais aucun livre de mathématiques (à l'exception d'un petit traité d'algorisme ${ }^{85}$ ), aucune table astronomique ni aucun manuel de base de la science des étoiles, comme le De sphera de Jean de Sacrobosco et le Liber introductorius d'Alcabitius, mentionnés tous deux comme tels par Simon de Phares dans la partie de son autobiographie relative à ses études à la faculté des arts de l'université de Paris, vers $1462-1465^{86}$. Voilà qui constitue un singulier contraste avec la seule véritable bibliothèque d'étudiant parisien analysée dans le corpus de Karine Rebmeister-Klein, celle de Jérôme Second, boursier du collège des Dix-Huit, mort en 1430, qui comprenait 9 manuscrits d'astronomie et d'astrologie, dont ces deux manuels, sur un total de 29 volumes ${ }^{87}$.

Autant dire qu'il semble rester peu de choses de ce que devait représenter la partie scientifique des « librairies » du collège de Maître Gervais à la fin du XV $v^{e}$ siècle. Certes, en 1501, le cardinal Georges d'Amboise se plaint, dans

81. Sur ce traité de Firmin de Beauval, achevé en 1338 mais qui comprend une table d'étoiles composée par Jean Vimond sur l'année de référence 1320 completo, soit 1321 incompleto $\left(\mathrm{BnF}\right.$, lat. $\left.7482, \mathrm{f}^{\circ} 61 \mathrm{v}^{\circ}-69 \mathrm{v}^{\circ}\right)$, et non pas sur l'année 1312 , comme l'indique l'édition incunable de 1485, voir L. THORNDIKE, A History of Magic and Experimental Science, vol. III, New York, 1934, p. 268-280, et Le Recueil, op. cit., p. 511-512.

82. Cette liste fragmentaire de $28 \mathrm{mss}$. a été publiée par E. Chatelain, Notice sur les manuscrits du collège de Maître Gervais, Paris, 1888, p. 15-16, et par K. ReBMEISTER-KLeIN, Les Livres des petits collèges, op. cit., t. II, p. 697-699.

83. Sur Bonatti, le plus fameux astrologue italien du XIII ${ }^{\mathrm{e}}$ siècle, voir L. Thorndike, $A$ History of Magic, op. cit., vol. II, New York, 1923, p. 825-835 ; M.-T. d'AlvernY, « Dante et les astrologues de son temps », Bulletin de la Société d'Études Dantesques du Centre Universitaire Méditerranéen, t. XIX (1970), p. 3-15 ; S. CAROTI, L'astrologia in Italia, Rome, 1983, p. 141-158, et en dernier lieu J.-P. BoudET, Entre science et "nigromance », op. cit., p. 83-85.

84. Sur l'astrologue anglais du XIv ${ }^{\mathrm{e}}$ siècle Jean d'Eschenden, alias John Eschenden ou Aschenden, et cet ouvrage intitulé également Summa anglicana, voir L. THORNDIKE, A History of Magic, op. cit., vol. III, p. 325-346 et 717-721 ; H. M. CAREY, Courting Disaster. Astrology at the English Court and University in the Later Middle Ages, Londres, 1992, p. 58-78, 188191, et N. WeILl-PARot, Les «Images astrologiques », op. cit., p. 406-409.

85. Copié par Jean Picot dans le ms. 192 de la Bibl. de l'Univ., fo 288-292, à la suite de la Vita Christi de Ludolphe le Chartreux.

86. Le Recueil, op. cit., p. 565-566. Sur ces manuels, voir L. Thorndike, The Sphere of Sacrobosco and its Commentators, Chicago, 1949, et AL-QABîsî (Alcabitius), The Introduction of Astrology. Editions of the Arabic and Latin Texts and an English Translation, C. BuRnetT, K. Yamamoto et M. Yano éd., Londres-Turin, 2004.

87. K. RebmeIster-Klein, Les Livres des petits collèges, op. cit., t. I, p. 144-146, et t. II, p. $565-568$. 
une lettre adressée à l'archidiacre de Bayeux, des vols récents de livres dont le collège a été la victime ${ }^{88}$, mais dans la première moitié $\mathrm{du} \mathrm{XVI}^{\mathrm{e}}$ siècle, l'institution semble prospère. En février 1529 , le nombre des grands boursiers passe de 12 à 15 , leurs bourses sont augmentées de six à huit sous parisis par semaine et celle des petits boursiers de trois à quatre sous. Parmi les grands boursiers, apparaît en cette occasion le nom d'Oronce Fine, ce qui constitue un complément intéressant à la biographie de ce grand savant, devenu peu après, en 1531, lecteur de mathématiques au Collège royal nouvellement créé par François I ${ }^{\text {er }}{ }^{89}$. Les choses se gâtent apparemment pour le collège de Maître Gervais à la fin du XVI siècle, époque à laquelle ses bourses semblent ne plus constituer que des sinécures: en 1598, la bourse de l'un des deux «mathématiciens », boursier depuis quinze ans et curé non résidant dans le collège depuis quatre années, est déclarée vacante, et son confrère astronome dispose de trois mois pour choisir entre sa bourse et une cure pour laquelle il a délaissé ses cours. L'institution des boursiers mathématiciens, très inférieure en prestige à celle des lecteurs au Collège royal qui paraît l'avoir largement vidée de sa substance, semble sur la voie de la décrépitude $^{90}$. Mais en 1612, la bibliothèque du collège de Maître Gervais est encore «belle, riche et magnifique», selon le témoignage de Jacques Du Breul, dans son Théâtre des antiquitez de Paris $^{91}$. En 1778, quelques années après sa fusion avec le collège Louis-le-Grand (1763), alors que les boursiers n'y sont plus qu'une douzaine, Louis XVI double le nombre total des bourses, qui passent de 24 à 48, et il confirme les lettres patentes de Charles V d'avril 1379, «ce faisant que le Grand-Aumônier de France continue, en qualité de Proviseur dudit Collège, de nommer aux bourses de cette fondation ${ }^{92} .12$ des 24 anciennes bourses sont affectées au diocèse de Bayeux, mais 12 des 24 nouvelles bourses sont réservées aux nobles de l'ensemble du royaume. Après quatre siècles d'existence, c'est le chant du cygne de l'institution créée par Gervais Chrétien.

88. E. Chatelain, Notice sur les manuscrits du collège de Maître Gervais, op. cit., p. 6.

89. Arch. de 1'Univ. de Paris, carton $n^{\circ} 21$, liasse 1, pièce $n^{\circ} 2,16$ fév. 1529 (n.s.) : ordonnance de Jean Le Veneur, grand aumônier de France, évêque de Lisieux et proviseur du collège. Sur Oronce Fine, voir D. Hillard et E. Poulle, «Oronce Fine et l'horloge planétaire de la Bibliothèque Sainte-Geneviève », Bibliothèque d'Humanisme et Renaissance, vol. XXXIII (1971), p. 311-334 ; E. Poulle, « Oronce Fine », Dictionary of Scientific Biography, C. C. GILlispie éd., New York, 1980, vol. XV, p. 153-157. Le cursus suivi par Oronce Fine se répéta un siècle plus tard pour Gilles Personne de Roberval qui, en 1632, enseignait la philosophie au collège de Maître Gervais et qui, le 24 juin 1634, remporta le concours pour la chaire de philosophie au Collège royal, antérieurement illustré par Ramus.

90. Arch. de l'Univ. de Paris, carton $\mathrm{n}^{\circ} 21$, liasse 1 , pièce $\mathrm{n}^{\circ} 26.2, \mathrm{f}^{\mathrm{o}} 6 \mathrm{v}^{\circ}-7,8-8 \mathrm{v}^{\circ}$ et 13 .

91. Cité par E. Chatelain, Notice sur les manuscrits du collège de Maître Gervais, op. cit., p. 5.

92. Déclaration du Roi, concernant le Collège de Maître-Gervais, réuni à celui de Louisle-Grand, donnée à Versailles, le 3 septembre 1778, et enregistrée au Parlement, le 7 septembre, Paris, P. G. Simon, 1779. 
Au total, le collège de Maître Gervais n'a donc jamais été un «colliege de astrologie et medicine », mais un établissement dominé qualitativement par les théologiens et quantitativement par les artiens, devenus «étudiants en humanités » à l'époque moderne. La création des deux bourses de scholares regis en 1377 visait à accorder une place secondaire, mais significative, à l'étude de la science des astres dans le cursus universitaire parisien, afin d'en faire une "science licite », assez étroitement contrôlée par le roi et l'Église. Quant à la création concomitante des deux bourses de médecins, elle relevait d'une tentative de rééquilibrage, à une époque où l'étude de la médecine n'était autorisée que dans une demi-douzaine de collèges parisiens sur 35. Un rééquilibrage cependant bien insuffisant : ce n'est naturellement pas grâce aux collèges qu'au moins un millier d'étudiants en médecine ont été formés par l'université de Paris dans les derniers siècles du Moyen Âge ${ }^{93}$. Le résultat, modeste, fut néanmoins incontestable, et l'on peut ainsi distinguer, au sein du collège de Maître Gervais, une coexistence harmonieuse entre la médecine et l'astronomie-astrologie, sans que ces deux disciplines y aient été clairement considérées comme les deux faces complémentaires d'une même profession. De ce point de vue, il est d'ailleurs bien possible qu'il y ait eu un certain écart entre le dessein initial de Charles V, celui de son premier médecin, et une institution dans laquelle «physiciens » et «astrologiens » vivaient sous un même toit mais étaient censés travailler dans deux « librairies » distinctes.

Jean-Patrice Boudet, Université d'Orléans, Faculté des Lettres, Langues et Sciences humaines, 10, rue de Tours, BP 46527, F-45065 Orléans Cedex 2.

\section{Charles V, Gervais Chrétien et les manuscrits scientifiques du collège de Maître Gervais}

Considéré par Simon de Phares et par certains historiens qui ont cru bon de lui faire confiance comme une institution axée principalement sur l'astrologie et la médecine, le collège fondé par le premier «physicien» de Charles V, Gervais Chrétien, en 1371, était en fait destiné en priorité, au départ, à des étudiants en théologie. Ce n'est qu'en 1377 qu'y furent instituées deux bourses de scholares regis, spécialisées dans «les sciences mathématiques licites », et deux bourses en médecine. Mais le rayonnement de l'activité de ces boursiers semble avoir été assez modeste et, autant qu'on puisse le savoir en consultant les sources subsistantes, notamment les manuscrits qui ont appartenu au collège de Maître Gervais et à certains de ses membres, cet établissement était voué bien davantage à l'étude de la théologie qu'à la médecine et au quadrivium.

Charles V - collège de Gervais Chrétien - astrologie - médecine - manuscrits scientifiques 


\section{Charles V, Gervais Chrétien and the Scientific Manuscripts of Maître Gervais' College}

Considered as an institution mainly devoted to astrology and medicine by Simon de Phares and some historians who believed that he was reliable, the college founded in 1371 by Charles V's first physician, Gervais Chrétien, was actually primarily dedicated to theological students. It was not before 1377 that were created there two bursaries for scholares regis, specialised in « licit mathematical sciences », and two medical fellowships. Yet the influence of the activity of these fellows seems to ave been rather moderate and - as far as we can learn from the material still extant, notably from manuscripts that belonged to Maître Gervais' College and to some of his members - this institution was devoted much more to theological studies than to medicine and quadrivium.

Charles V - Gervais Chrétien's College - astrology - medicine - scientifical manuscripts 